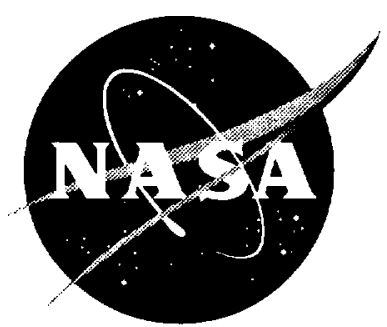

\title{
Autonomous Modal Identification of the Space Shuttle Tail Rudder
}

Richard S. Pappa

Langley Research Center, Hampton, Virginia

George H. James III and David C. Zimmerman

University of Houston, Houston, Texas

June 1997

National Aeronautics and

Space Administration

Langley Research Center

Hampton, Virginia 23681-0001 


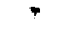




\title{
AUTONOMOUS MODAL IDENTIFICATION OF
}

\author{
THE SPACE SHUTTLE TAIL RUDDER
}

\author{
Richard S. Pappa \\ Structural Dynamics Branch \\ NASA Langley Research Center \\ Hampton, Virginia 23681 \\ Email: r.s.pappa@larc.nasa.gov
}

\author{
George H. James III and David C. Zimmerman \\ Mechanical Engineering Department \\ University of Houston \\ Houston, Texas 77204 \\ Email:ghjames@mainsail.me.uh.edu \\ dzimmerman@uh.edu
}

\begin{abstract}
Autonomous modal identification automates the calculation of natural vibration frequencies, damping, and mode shapes of a structure from experimental data. This technology complements damage detection techniques that use continuous or periodic monitoring of vibration characteristics. The approach shown in the paper incorporates the Eigensystem Realization Algorithm (ERA) as a data analysis engine and an autonomous supervisor to condense multiple estimates of modal parameters using ERA's Consistent-Mode Indicator and correlation of mode shapes. The procedure was applied to free-decay responses of a Space Shuttle tail rudder and successfully identified the seven modes of the structure below $250 \mathrm{~Hz}$. The final modal parameters are a condensed set of results for 87 individual ERA cases requiring approximately five minutes of CPU time on a DEC Alpha computer.
\end{abstract}

KEYWORDS: Modal testing, vibration testing, autonomous modal identification, damage identification using vibration testing, Eigensystem Realization Algorithm.

\section{INTRODUCTION}

Modal identification is the process of calculating natural frequencies, damping, and mode shapes (modal parameters) from experimental data. In most cases the experimental data are free-decay vibration measurements, frequency response functions (FRFs), or impulse response functions (IRFs) obtained by inverse Fourier transformation of FRFs or by using the Observer-Kalman Filter Identification (OKID) technique (Juang, 1994). The Eigensystem Realization Algorithm (ERA) identifies structural modal parameters from IRFs or free-decay data. It is a multiple-input/multipleoutput, time-domain technique that efficiently identifies many modes simultaneously including closely spaced and weakly excited modes. Since its introduction in 1984, ERA has been used effectively on a large number of structures, and many publications discuss both the theoretical and practical aspects of the method (Pappa, 1997a).

ERA often generates good results using the default values of various algorithm parameters. However, depending on accuracy requirements, improvement of results may be necessary or desirable. One approach to improving modal identification results is to conduct additional tests. Weakly excited or weakly observed modes may be identified better by increasing excitation amplitudes, changing the number or orientation of exciters, improving signal-to-noise ratios of the data by different filtering, windowing, or averaging methods, adding or moving sensors, adjusting amplifier settings, etc. An entirely different approach is to perform additional ERA analyses of the same data set(s) using other values of the algorithm parameters. For example, the data record length may be increased to improve the accuracy of linear, lightly damped, low-frequency modes (probably at the expense of other modes with higher nonlinearity, damping, or frequency). The extent to which modal identification results fluctuate with changes in algorithm parameters depends on the dynamic complexity of the structure, notably its modal density and degree of nonlinearity, and on the quality of the experimental measurements.

Autonomous modal identification of structures is a new technology in its early stages of development. In this paper, only the second approach for improving identification results is addressed; that is, the improvement of identified modal parameters by performing additional ERA 
analyses of existing data set(s). In a previous study, autonomous modal identification was implemented using sliding-bandwidth filtering and both single-input and multiple-input analyses (Pappa et al, 1997b). In the present study, the size of the ERA Hankel data matrix and the assumed number of modes are varied. These are two additional, principal algorithm parameters. As more experience is gained with autonomous modal identification, a larger number of parameters will be included in the procedure. A full implementation will eventually incorporate all ERA algorithm parameters.

In recent years, considerable attention has been given to the topic of damage detection of structures using vibration measurements (Doebling et al, 1996; Flett, 1996; Grygier, 1994; James, 1996; Zimmerman et al, 1996). This technology applies to a wide range of structures including spacecraft, aircraft, buildings, bridges, off-shore oil platforms, and wind turbines. Many of the methods under development detect damage by monitoring changes in modal parameters. Using modal parameters for this purpose is attractive for several reasons, including 1) modal parameters change (to some degree) with any change in the mass, stiffness, or damping of the structure, 2) damage at inaccessible locations can be detected by changes in modal parameters measured at accessible locations, and 3 ) modal identification technology is widely used in laboratories and a broad experience base exists. The capability of generating structural modal parameters autonomously (i.e., without human involvement) for damage detection is the primary motivation of the work discussed in this paper.

The next section of the paper describes the test article for this study, and the manner in which it was tested in the laboratory. The following section summarizes the autonomous modal identification technique. For this application, three sets of ERA analyses are performed comprising a total of 87 cases followed by a final, overall mode condensation. The remainder of the paper shows principal identification results including a summary of the final, condensed set of modal parameters.

\section{TEST ARTICLE AND TEST DESCRIPTION}

Figure 1 shows the rudder/speed brakes (RSBs) of the Space Shuttle Orbiter. There are two pairs of RSBs, an upper pair and a lower pair. Each pair contains left and right surfaces that rotate in the same direction when used as a rudder and in opposite directions when used as a speed brake. The surfaces rotate up to plus or minus 27 degrees as a rudder, individually up to 49 degrees as a speed brake, or combined for joint rudder/speed brake control. The vertical tail structure is covered with reusable thermal insulation and is designed to withstand a 163 decibel acoustic environment with a maximum temperature of 350 degrees $F$.

The structure used in this study is shown in Fig. 2. It is an upper-right RSB, and was tested with free-free boundary conditions by suspending it from the actuator attach points using springs and cables. The test occurred at the NASA Johnson Space Center (JSC) in December 1996. This structure is one component of a circa-1980 Vertical Stabilizer Assembly (VSA) being used at JSC and the University of Houston for finite-element model correlation and damage identification studies. The VSA originally served as an acoustic and static fatigue test article. The components of the VSA are being tested individually and assembled to develop techniques for correlating component and assembled models. The structure will then be damaged in various ways to test damage identification methods. The RSB is approximately 48 " $x 7$ " $\times 120$ " in size, and weighs $180 \mathrm{lbs}$.

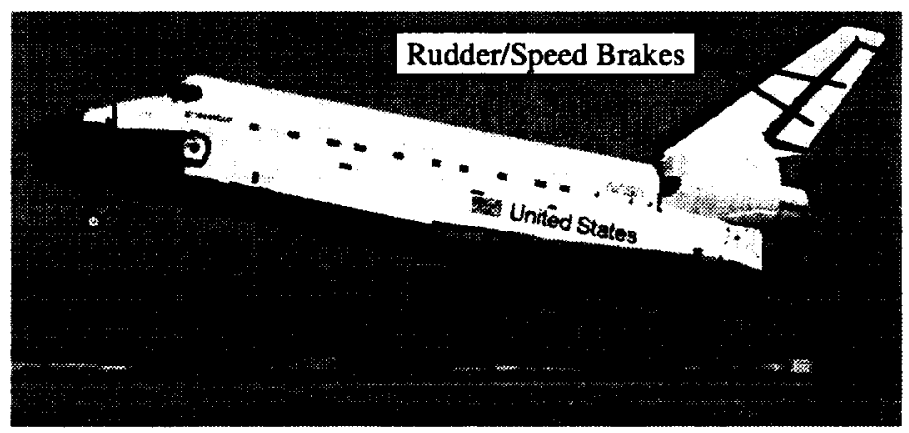

Fig. 1. SPACE SHUTTLE ORBITER

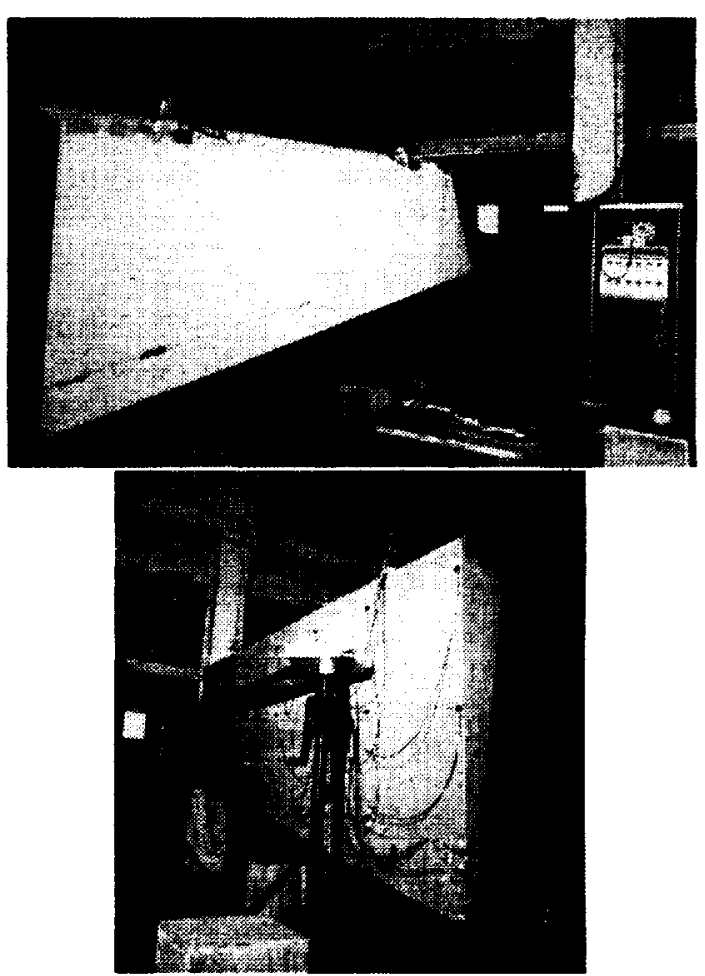

Fig. 2. MODAL TEST CONFIGURATION 
Figure 3 shows the excitation and response degrees-of-freedom used in the modal test. The structure was excited several times at each of the 7 locations shown in Fig. 3(a) with a forceinstrumented hammer, and frequency response functions (FRFs) were calculated between the applied forces and each of the 47 accelerometers shown in Fig. 3(b). Note that the majority of impacts and response measurements are in the

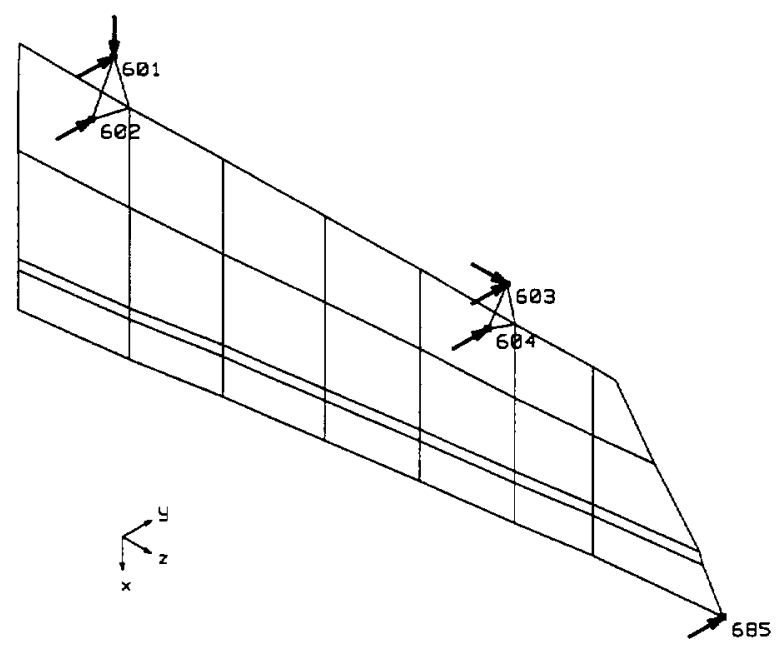

(a) 7 Impact Locations $y$ direction (perpendicular to the surface of the structure) which emphasizes the primary, out-ofplane bending and torsional modes of the structure. In addition to calculating FRFs, the free-decay vibration responses for the final impact at each excitation location were also recorded. The autonomous modal identification will analyze these free-decay responses.

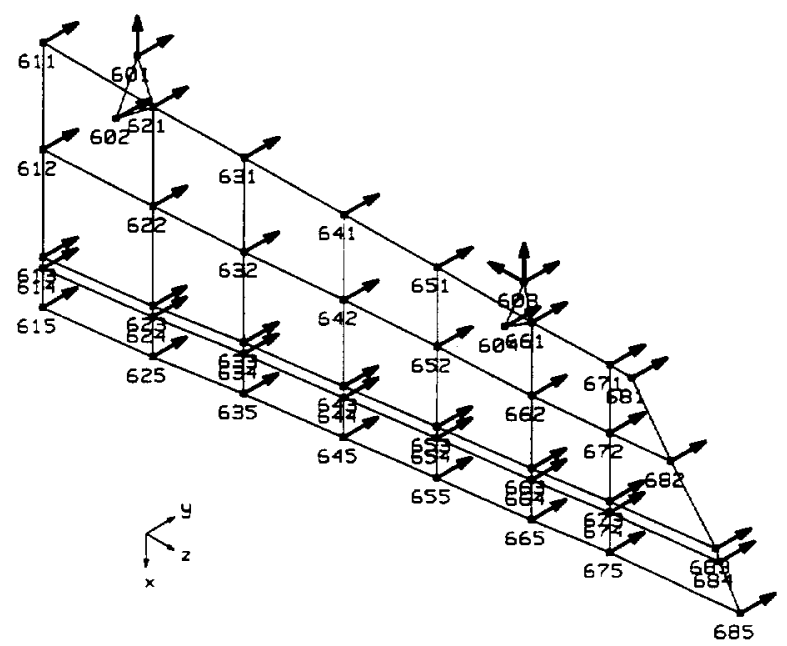

(b) 47 Accelerometers

Fig. 3. EXCITATION AND RESPONSE LOCATIONS
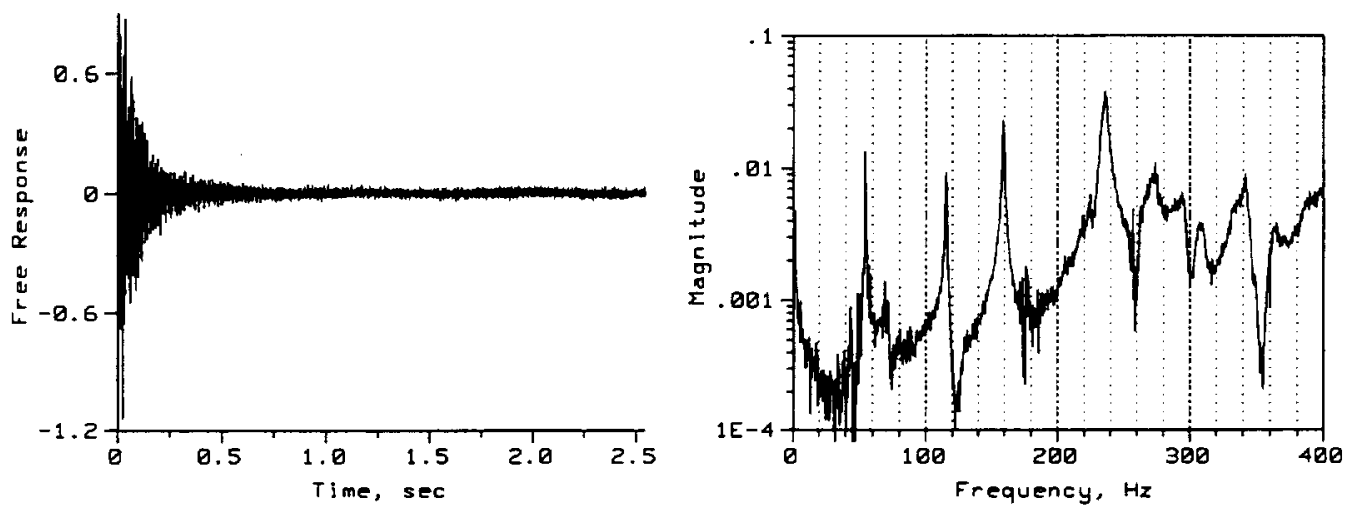

(a) Excitation: 603y, Response: 672y
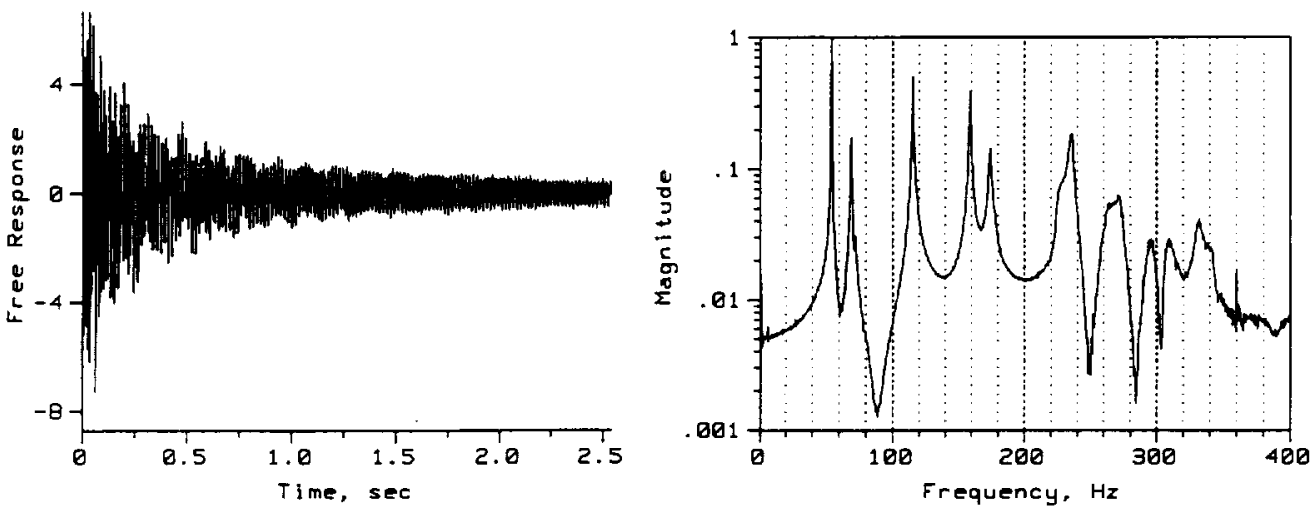

(b) Excitation: 685y, Response: 611y

Fig. 4. TYPICAL FREE-DECAY RESPONSES AND SPECTRA 
Typical free-decay measurements and their corresponding FFT-magnitude spectra appear in Fig. 4. Each time history contains 2033 data points at a sampling rate of $800 \mathrm{samples} / \mathrm{sec}$, and the data is alias-free out to a frequency of 312.5 $\mathrm{Hz}$. The relatively noisy data in $\mathrm{Fig} .4(\mathrm{a})$ is representative of most measurements obtained using $y$-direction excitation at the actuator attach points (points 601-604). The relatively cleaner data in Fig. 4(b) is representative of most measurements obtained using $y$-direction excitation at the apex of the structure (point 685). Although not shown, most measurements obtained using $x$ and $z$-direction excitation at points 601 and 603 , respectively, are even noisier than that shown in Fig. 4(a). The autonomous modal identification will analyze all 329 response measurements simultaneously to obtain global estimates of the modal parameters $(329$ responses $=47$ accelerometers $\times 7$ impact locations). By using all data simultaneously in the analysis, the cleaner measurements tend to dominate in the calculations over the noisier measurements because of their larger amplitudes.

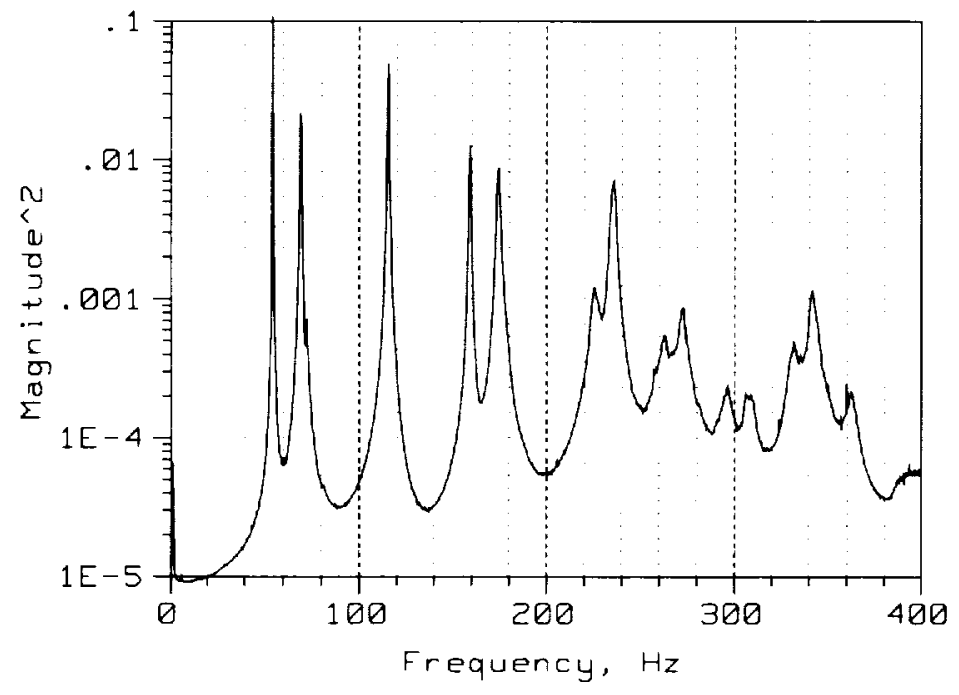

Fig. 5. AVERAge POWER SPECtRUM

Figure 5 shows the average power spectrum of all 329 free-decay responses. It provides a good overview of the natural frequencies of the structure and their relative strengths in the measurements. This function is the average squared magnitude of all FFT spectra such as shown on the righthand sides of Figs. 4(a) and 4(b). It indicates that there are seven predominant modes of the structure from zero to $250 \mathrm{~Hz}$ (the frequency range of interest). There is also a small inflection in Fig. 5 at $72 \mathrm{~Hz}$ indicating an additional mode in this frequency range, but it is too poorly excited and/or too poorly sensed to be accurately identified. (This peak at $72 \mathrm{~Hz}$ is more apparent in other individual spectra not shown.) This mode is probably a local mode of the cable suspension system.

\section{AUTONOMOUS MODAL IDENTIFICATION}

Figure 6 is a flow diagram of the autonomous modal identification approach. There are four principal steps: 1) running the Eigensystem Realization Algorithm (ERA) (Pappa, 1997a), 2) applying thresholds to the results which filter out unreasonable values, 3 ) condensing (i.e., reconciling) the most-recent set of candidate modal parameters (the output of the thresholding step) with results obtained in previous passes around the feedback loop shown in Fig. 6, and 4) selecting appropriate algorithm parameters for the next ERA analysis. Note that the Mode Condensation block is also known as the Supervisor because it is responsible for determining how multiple ERA analyses are combined into a coherent, overall set of results. The following paragraphs discuss each of the four steps in more detail. The dashed line in Fig. 6 from the Supervisor to the Case Selector indicates a desirable feature that is under development, but it is not yet available. The final set of modal parameters is the last-computed output of the mode condensation process.

\section{ERA}

The Eigensystem Realization Algorithm serves as a data analysis "engine." ERA is a wellestablished, documented procedure for estimating structural modal parameters (natural frequencies, modal damping, and mode shapes) from free-decay vibration responses (or impulse response functions) based on a set of user-specified algorithm parameters (Pappa, 1994). Although

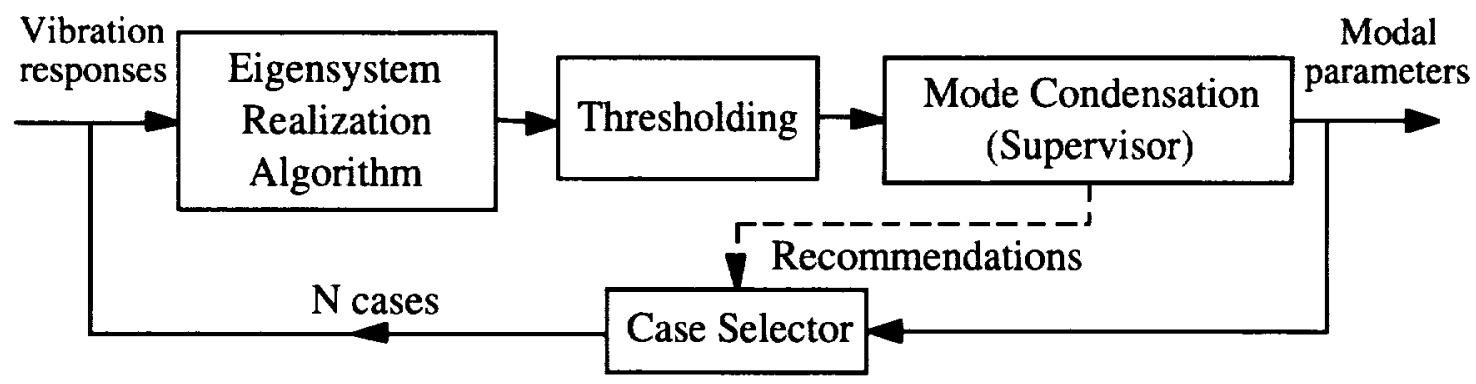

Fig. 6. AUTONOMOUS MODAL IDENTIFICATION APPROACH 
default values and/or guidelines are available for each algorithm parameter, users have considerable freedom in selecting their values. With experimental data, the ERA results will fluctuate to some degree with any change in the algorithm parameters. The magnitude of fluctuation varies from mode to mode, and it depends in a complex manner on the characteristics and quality of the data, including its modal density, degree of nonlinearity, noise level, modal response amplitude, damping level, spatial linear independence of closely spaced modes, data sampling frequency, etc.

In addition to generating modal parameters, ERA also calculates various "accuracy indicators" associated with each result. The principal ERA accuracy indicator is the Consistent-Mode Indicator (CMI) which provides a single, overall value for each mode ranging from zero to $100 \%$ (Pappa et al, 1993). Modes with CMI values greater than approximately $80 \%$ are identified with high confidence. Modes with values from $80 \%$ to $1 \%$ display moderate to large uncertainty. Extraneous "noise modes" have CMI values of approximately zero. The CMI calculation uses both the temporal and spatial consistency of the results, and it is a robust, proven indicator of modal identification accuracy.

\section{Thresholding}

The thresholding step in Fig. 6 eliminates unfeasible modal identification results generated by ERA. A normal characteristic of ERA is that it generates extra, nonphysical modes in addition to the true, structural modes. The "noise modes" mentioned in the previous paragraph usually comprise a large fraction of these nonphysical modes. For good results with experimental data, at least half of the total number of identified modes will normally be nonphysical modes. Currently, nonphysical modes are eliminated from the results using the simple criteria shown in Fig. 7. Note that the CMI cutoff of $50 \%$ will allow some real, structural modes with poor accuracy to be deleted, but this is acceptable in most cases. (Hopefully, that mode is identified better in another ERA analysis.) The damping and frequency cutoffs of $10 \%$ and $1 \%$, respectively, are good selections for typical engineering structures having modal damping ratios on the order of $2 \%$ or less. These values would have to be increased for structures with higher damping.

\section{Thresholding}

Delete modes with:

$\mathrm{CMI}<50 \%$, Damping $<0$ or $>10 \%$, or Frequency within $1 \%$ of edges of analysis bandwidth.

\section{Fig. 7. THRESHOLDING LOGIC}

\section{Mode Condensation (Supervisor)}

Figure 8 shows the mode condensation logic, which is presently the principal autonomous aspect of the procedure. Mode condensation selects the best estimate of each structural mode from
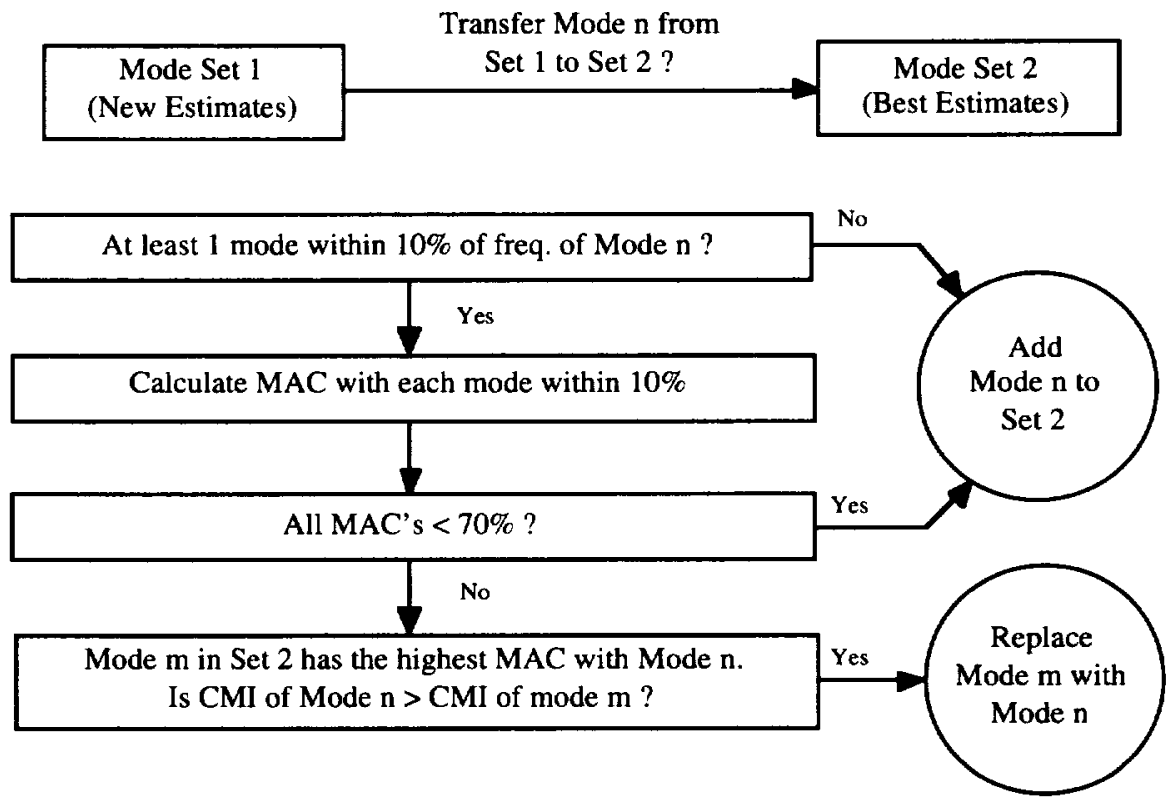

Fig. 8. MODE CONDENSATION LOGIC 
multiple estimates of the mode. In other words, given a set of best estimates of the modal parameters (a set which is available initially after the first pass through the loop of Fig. 6), mode condensation decides whether or not new estimates of the modal parameters (referred to as Set 1 in Fig. 8) will be added to or replace results already in the best set (referred to as Set 2). steps:

Mode condensation consists of the following

1. For each mode in Set 1 (Mode $n$ ), compare its frequency with the frequencies of all modes in Set 2 . If there are no modes of Set 2 within $10 \%$ in frequency of Mode $n$, add Mode $n$ to $S$ et 2 . Recall that Mode $n$ has already passed through the Thresholding step, so it is probably a true, structural mode (albeit with some inaccuracy if its CMI value is not high).

2. For each mode of Set 2 within $10 \%$ in frequency of Mode $n$, calculate the Modal Assurance Criterion (MAC) (Pappa, 1994, p.113). MAC is the square of the correlation coefficient of the two modal vectors (mode shapes) and measures the similarity of mode shapes. If all MAC values are less than $70 \%$, add Mode $n$ to Set 2 because its mode shape is significantly different than all modes in Set 2 with similar frequency.

3. If there are one or more MAC values of $70 \%$ or greater, select the mode in Set 2 having the highest MAC (Mode $m$ ), and compare its CMI value with the CMI of Mode n. If the CMI of Mode $n$ (the new mode) is greater than that of Mode $m$, replace Mode $m$ in Set 2 with Mode $n$.

As mentioned earlier, information generated during the mode condensation activity will eventually be used to recommend algorithm parameters for subsequent ERA analyses (indicated by the dashed line in Fig. 6). A large amount of information is obtained during mode condensation concerning the characteristics of the data (such as the linear independence of closely spaced modes), and this information could be used to select better algorithm parameters. This capability is under development and is not yet available.

\section{Case Selector}

The final block in Fig. 6 is the Case Selector. Because the rudder/speed brake test article is a relatively simple structure, the case selection logic shown in Fig. 9 proved to be adequate for this application. Set $A$ is a standard, default type of initial ERA analysis. Although it involves 35 cases, the total execution time is quite short (approximately $75 \mathrm{secs}$ ) because the Hankel matrix and the number of measurements are fairly small. The final results of Set $A$ (discussed in the next section of the paper) contained 2 modes with CMI values less than $90 \%$ (out of a total of 7 modes below $250 \mathrm{~Hz}$ ). Accordingly, Set $B$ was then executed in an attempt to improve the accuracy of these modes. After both Sets A and B completed, Set $C$ combined the two groups of results into a final, overall set of results using the mode condensation logic of Fig. 8.

\section{Case Selection}

Set $A$ (35 cases): $47 \times 7$ MIMO analysis with Hankel matrix of 470 rows $x 70$ cols, Assumed No. of Modes - 1 through 35.

Stop here if all modes in the final results of Set $\mathrm{A}$ have $\mathrm{CMI}>=90 \%$. If not, proceed to Sets $B$ and $C$.

Set $B$ (52 cases): $47 \times 7$ MIMO analysis with Hankel matrix of 470 rows $x 105$ cols, Assumed No. of Modes - 1 through 52.

Set C: Overall Results - Combine results of Sets A and B by mode condensation.

\section{Fig. 9. CASE SELECTOR LOGIC}

Sets $A$ and $B$ are multiple-input/multipleoutput (MIMO) analyses using all 329 free-decay response measurements simultaneously. Both sets use a Hankel data matrix with 470 rows $(10$ shifts of the 47 outputs). However, the number of columns in the matrix increases from 70 in Set $A$ (10 shifts of the 7 inputs) up to 105 in Set B (15 shifts of the 7 inputs). The maximum number of identified modes in any ERA analysis is always equal to one-half the minimum dimension of the Hankel matrix. For good results with experimental data, the maximum number of identified modes is typically in the range of approximately 2 to 10 times the number of structural modes in the data bandwidth (up to $400 \mathrm{~Hz}$ in this problem). The numbers of columns in Sets A and B (70 and 105) were chosen based upon an estimated number of structural modes from zero to $400 \mathrm{~Hz}$ of approximately 15 obtained by counting the peaks in the average power spectrum, Fig. 5 .

\section{MODAL IDENTIFICATION RESULTS}

\section{Natural Frequencies and CMI}

Figures $10(\mathrm{a})$ and $10(\mathrm{~b})$ show the ERAidentified natural frequencies between zero and 250 $\mathrm{Hz}$ (the frequency range of interest) as a function of the assumed number of modes for $S$ ets $A$ and $B$, respectively. There are seven predominant modes of the structure in this frequency range as indicated. Each row of results in Fig. 10 corresponds to a separate ERA analysis with the 


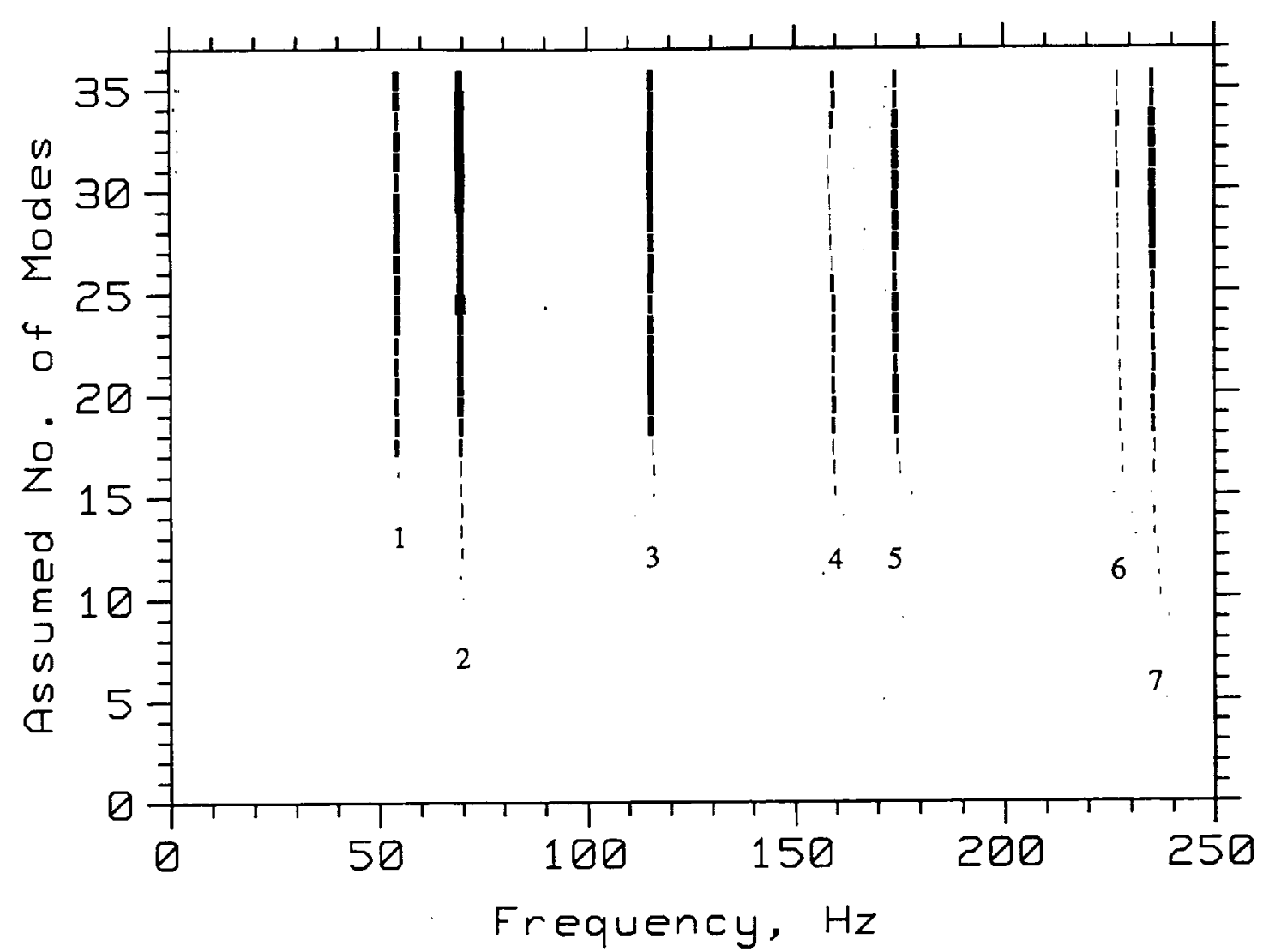

(a) Set A (35 cases): Hankel Matrix Size - 470 rows $x 70$ columns

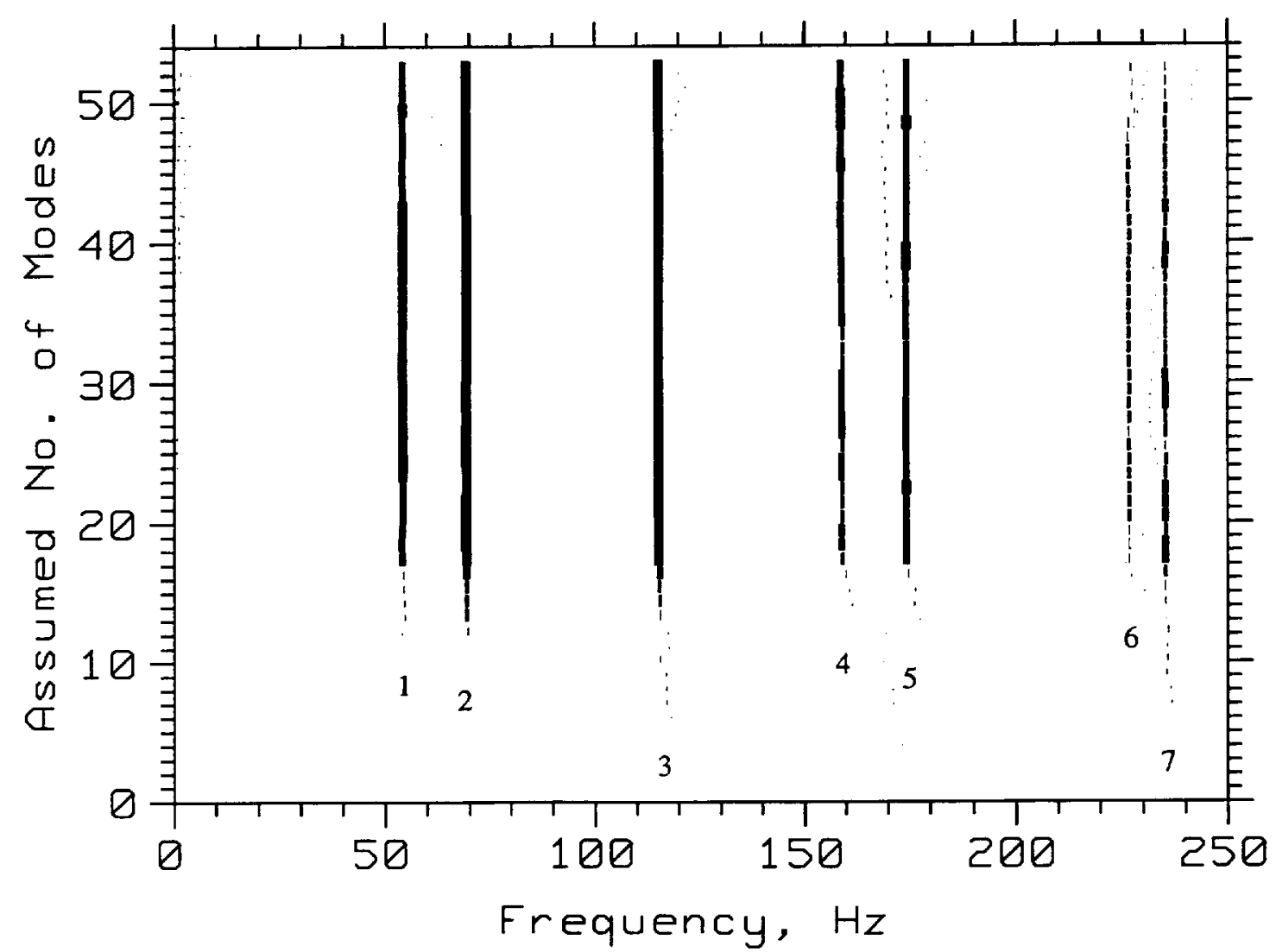

(b) Set B (52 cases): Hankel Matrix Size - 470 rows x 105 columns

Fig. 10. IDENTIFIED FREQUENCIES VS. ASSUMED NUMBER OF MODES 
specified number of assumed modes. Each identified mode is represented by a short vertical dash at the associated frequency. The height and width of each dash express the confidence of the result based on the Consistent-Mode Indicator (CMI) (Pappa et al, 1993), as described in the following paragraph. (Note: These natural frequency results occur prior to the Thresholding block of Fig. 6. For clarity, modes with CMI values less than $1 \%$ are not shown.)

The height of each vertical dash in Fig. 10 is directly proportional to the CMI value such that a mode with a value of $100 \%$ has a vertical dash height equal to the distance between $y$-axis tic marks. The width of each dash also increases with increasing CMI. This is done by using four line widths ranging from narrowest to widest for the following ranges of CMI: 1) below $80 \%$, 2) from $80 \%$ to $90 \%, 3$ ) from $90 \%$ to $95 \%$, and 4 ) above $95 \%$. (On a computer terminal, the four line widths correspond to four different colors, rather than line widths, to allow quick overview of the results.)

In this application, the CMI accuracy indicator did an excellent job distinguishing the true, structural modes from the nonphysical, computational modes generated by ERA. Figures 11 (a) and 11(b) show typical results from Sets $A$ and $B$, respectively. The seven structural modes have CMI values that are consistently much higher than those of the nonphysical modes. The majority of nonphysical modes, in fact, have values less than $1 \%$. The unusually high values near $0 \mathrm{~Hz}$, and at 120 and $240 \mathrm{~Hz}$, are due to small DC offsets in the data and electrical noise, respectively. The other unusually high value at approximately 170 $\mathrm{Hz}$ is probably a local mode of the cable suspension system.

Although CMI clearly distinguishes the seven structural modes from the nonphysical modes, a close examination of Fig. 10 shows considerable variations of CMI for most of the structural modes as a function of the assumed number of modes (indicated by varying line widths). Out of the 14 structural-mode results shown in Fig. 10(a,b), only Modes 2 and 3 in Fig. $10(\mathrm{~b})$ have uniformly increasing CMI with increasing numbers of assumed modes. This desirable, convergent behavior occurs for well-excited, linear modes when the Hankel matrix size is "large enough." On the other hand, all of the other results in Fig. 10 display fluctuating CMI values as the assumed number of modes increases. Unfortunately, such fluctuations are the rule rather than the exception with many experimental data sets due to unavoidable "real world" effects, particularly nonlinearity and noise. The thresholding and mode condensation logic discussed earlier in the paper are designed to sift through the results of these 87 cases and extract the most-confident set of structural modal parameters.

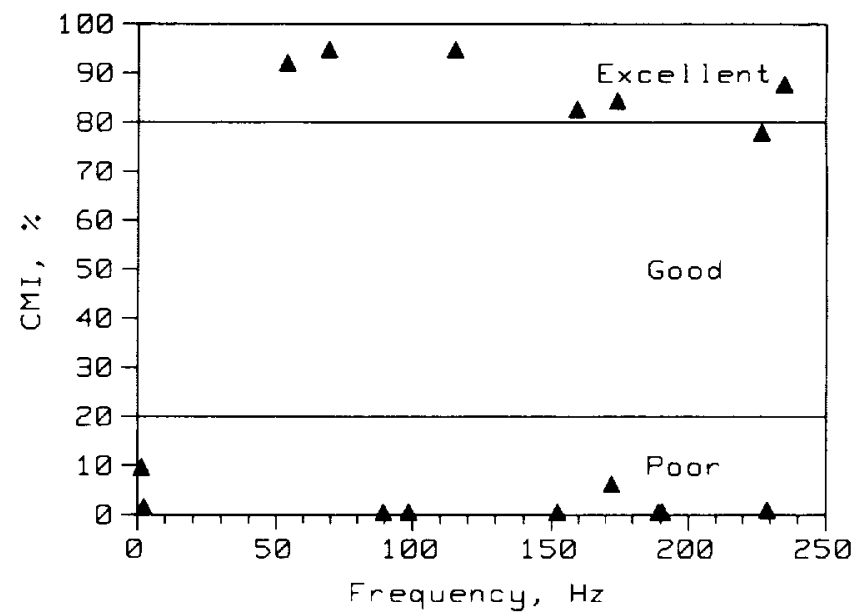

(a) Set A: Top Row of Fig. 10(a)

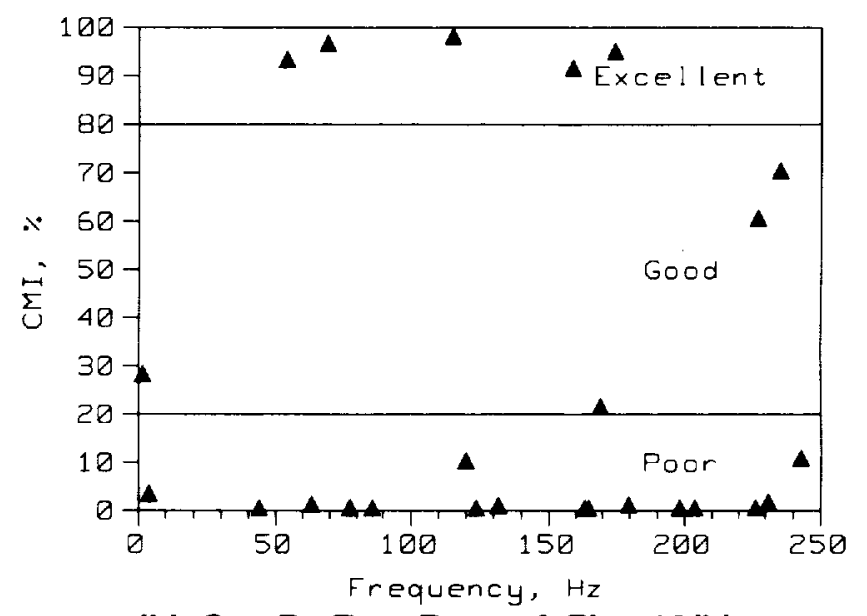

(b) Set B: Top Row of Fig. 10(b)

\section{Fig. 11. TYPICAL DISTRIBUTIONS OF CMI VALUES}

\section{Mode Condensation Results}

As indicated in Fig. 6, the final set of modal parameters from the autonomous modal identification approach is the output of the Mode Condensation process at the end of each case. If a sufficient collection of cases is run, the final, condensed set of modal parameters will be the best estimates of the true, structural modes (and nothing else).

This autonomous modal identification approach performed extremely well in this application. Figure 12 sshows the number of identified modes (i.e., the number of modes in the final, condensed set of results) at the end of each case for both Sets $A$ and B. Recall from Fig. 9 that condensed results are obtained individually for Sets $A$ and $B$, and then the individual results are combined in Set $C$ using a final mode condensation. The dips in Fig. 12 show that modes are being replaced according to the logic of Fig. 8. At each abscissa value, the 
larger of the two numbers is the total number of modes in Set 2 at the completion of the case. The smaller of the two numbers is the number of modes retained from the previous case. In other words, the difference between the two numbers is the number of modes replaced as a result of the ERA analysis for the particular Case Number. The total number of identified modes in both Sets $A$ and $B$ steadily increases up to the true value of seven, with many fluctuations along the way indicating continuous improvement of results. This convergent behavior with continuous improvement of the results is precisely the way in which autonomous modal identification is designed to operate.

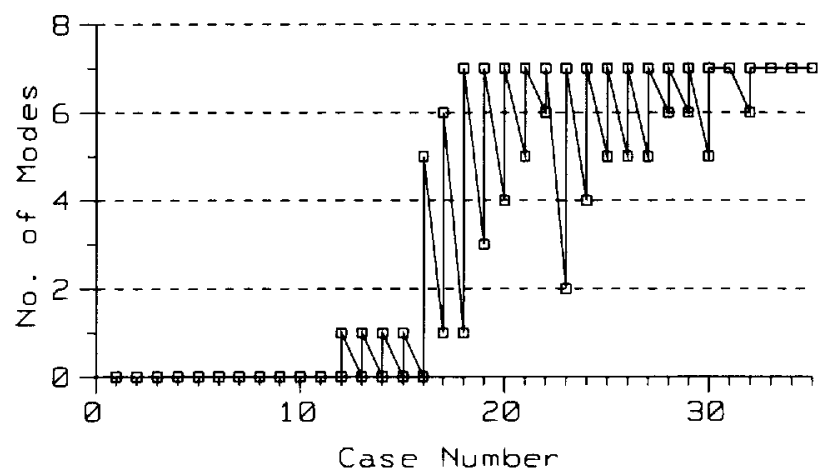

(a) Set A (35 cases)

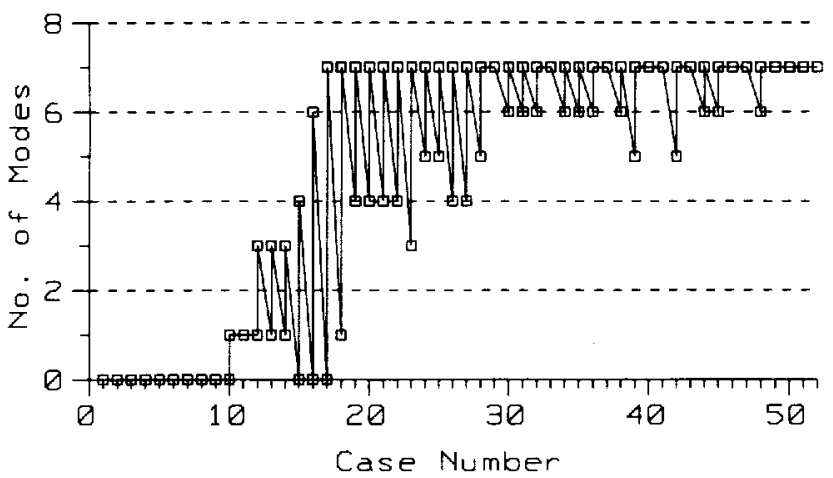

(b) Set B (52 cases)

Fig. 12. NUMBER OF IDENTIFIED MODES VS. CASE NUMBER

\section{Final Modal Identification Results}

The final set of results for both Sets $A$ and $B$ consisted of estimates of the seven true, structural modes. Note in Fig. 12 that both Sets converged to a stable number of modes (i.e., the replacement of modes slowed down and then ceased entirely as the Case Number approached 35 in Set $A$ and 52 in Set B). Following completion of Sets A and B, Set C executed a final mode condensation on these two individual sets of results.

Table 1 shows these final, overall modal identification results obtained in Set $C$. The second column in the table gives the Case No. from which the result was obtained. Note that 6 of the 7 modes came from Set B which used a larger Hankel data matrix size than $S$ et $A$. In many cases, though not always, increasing the size of the ERA Hankel matrix will increase modal identification accuracy. However, there is a disproportionate increase in the computation time. For example, in this application Set A required 75 secs of CPU time on a DEC Alpha 4000 computer, whereas $S$ et $B$ required 213 secs of CPU time but achieved higher accuracy in many of the modes.

All of the CMI values in Table 1 are greater than $80 \%$ indicating high confidence in the results. Furthermore, five of the CMI values are above $95 \%$ indicating extremely high confidence in these modes. The righthand column in Table 1 also gives the Weighted Modal Phase Collinearity (MPC-W) which is a parameter used in the CMI calculation (Pappa, 1993). MPC-W measures the nearness of the mode shape to a monophase vector (i.e., to a classical, normal mode). Values greater than $99 \%$ are extremely high.

Figure 13 shows the corresponding mode shapes. All seven of the modes are global, out-ofplane bending and torsional modes as expected. Each mode has a well-defined, predictable shape, substantiating the accuracy of the results. Furthermore, all seven frequencies and mode shapes agree closely with another, independent set

Table 1. FINAL MODAL PARAMETERS AND ACCURACY INDICATORS (SET C)

\begin{tabular}{|c|c|c|c|c|c|}
\hline $\begin{array}{c}\text { Mode } \\
\text { No. }\end{array}$ & $\begin{array}{c}\text { Case } \\
\text { No. }\end{array}$ & $\begin{array}{c}\text { Frequency, } \\
\mathrm{Hz}\end{array}$ & $\begin{array}{c}\text { Damping } \\
\text { Factor, \% }\end{array}$ & $\begin{array}{c}\text { CMI, } \\
\%\end{array}$ & $\begin{array}{c}\text { MPC-W, } \\
\%\end{array}$ \\
\hline \hline 1 & $31-$ Set B & 54.3 & 0.17 & 97.24 & 99.94 \\
\hline 2 & $44-$ Set B & 69.3 & 0.59 & 98.52 & 99.99 \\
\hline 3 & $26-$ Set B & 115.3 & 0.35 & 98.77 & 99.94 \\
\hline 4 & $45-$ Set B & 158.8 & 0.28 & 96.13 & 99.92 \\
\hline 5 & $48-$ Set B & 174.6 & 0.73 & 95.53 & 99.58 \\
\hline 6 & 42 - Set B & 226.6 & 1.12 & 87.61 & 98.68 \\
\hline 7 & 28 - Set A & 235.3 & 0.61 & 91.96 & 95.89 \\
\hline
\end{tabular}




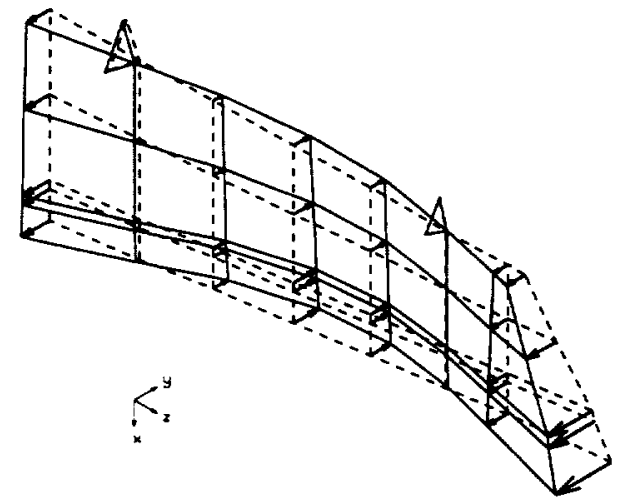

(a) First Bending at 54.3 Hz

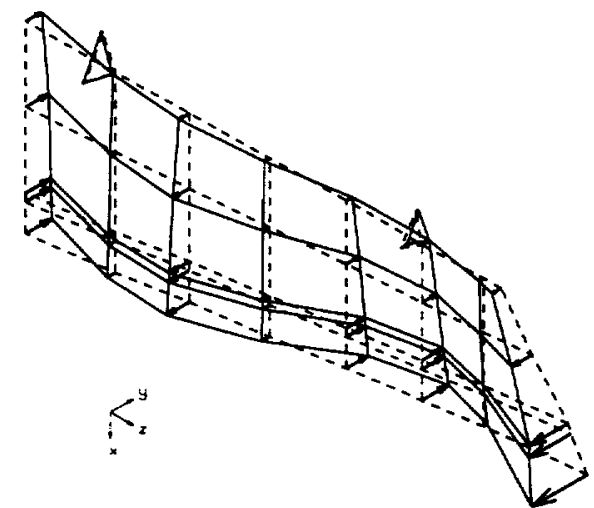

(c) Second Bending at $115.3 \mathrm{~Hz}$

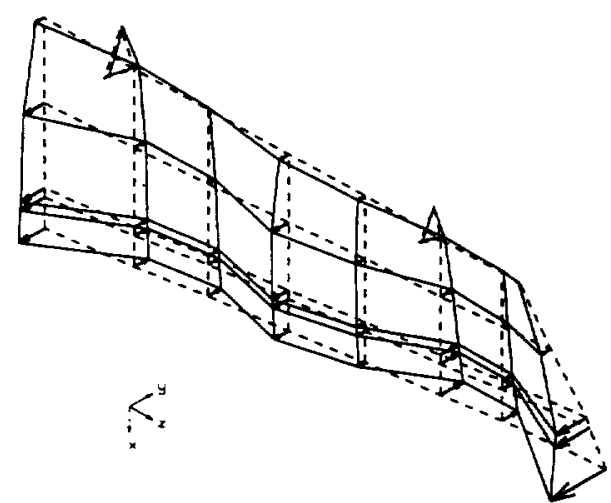

(e) Third Bending at $174.6 \mathrm{~Hz}$

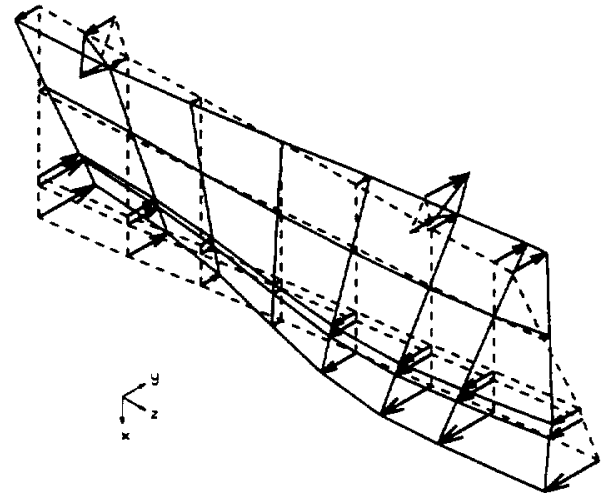

(b) First Torsion at $69.3 \mathrm{~Hz}$

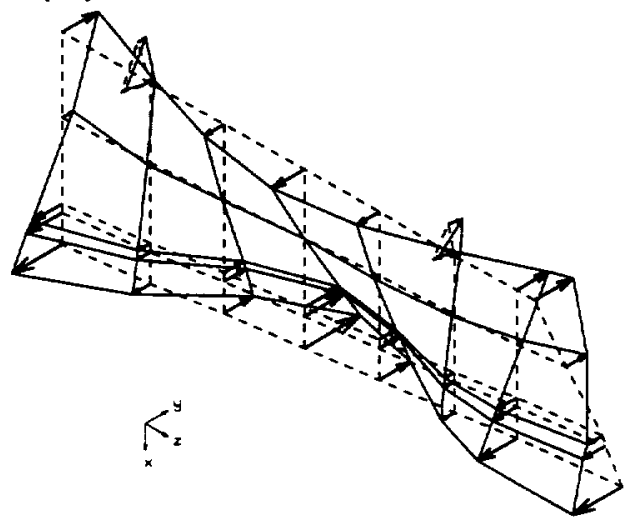

(d) Second Torsion at $158.8 \mathrm{~Hz}$

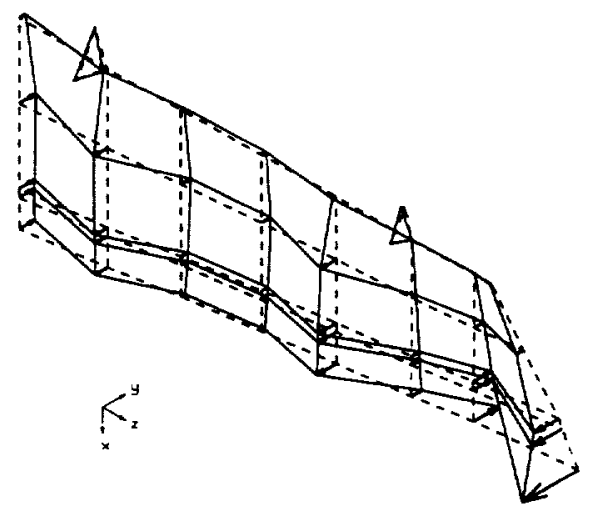

(f) Fourth Bending at $226.6 \mathrm{~Hz}$

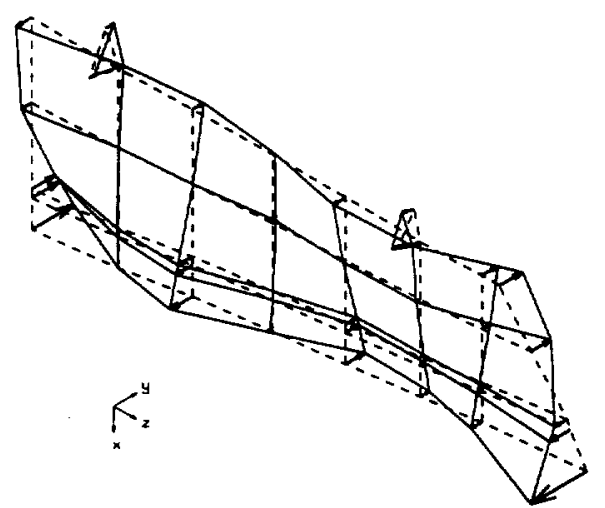

(g) Third Torsion at $235.3 \mathrm{~Hz}$

Fig. 13. MODE SHAPES (SET C: FINAL RESULTS) 
of results obtained by analyzing frequency response functions with the Polyreference technique. Good correlation of the two independent sets of results provides additional confidence in the accuracy of the autonomous modal identification approach described in this paper.

\section{CONCLUSIONS}

Considerable research is currently underway on several fronts in the area of damage detection of structures using changes in vibration characteristics. Many of the techniques under development require periodic calculation of structural modal parameters from experimental data. An attractive approach to this problem is autonomous modal identification. This new technology also promises improved speed and accuracy for more-traditional laboratory applications of modal identification techniques.

The autonomous modal identification approach described in the paper is a fairly straightforward extension of the Eigensystem Realization Algorithm. It successfully identified the seven vibration modes of the Shuttle Orbiter tail rudder from zero to $250 \mathrm{~Hz}$ in approximately five minutes of computer time. As more experience is gained using this technology on a variety of other structures, additional logic and features will be added to the software to improve its performance and reliability in generating optimum modal parameters without human involvement.

\section{ACKNOWLEDGMENTS}

The authors acknowledge the advice and assistance of Mike Grygier of the Johnson Space Center who serves as NASA technical monitor for the Vertical Stabilizer Assembly test program.

\section{REFERENCES}

Doebling, S. W., Farrar, C. R., Prime, M. B., and Shevitz, D. W., 1996, "Damage Identification and Health Monitoring of Structural and Mechanical Systems from Changes in Their Vibration Characteristics: A Literature Review," Los Alamos Report LA-13070-MS.

Flett, M., 1996, “Damage Detection Using System Identification Techniques," Website: http://nceer. eng. buffalo.edu/bibs/dam det ec. ht ml.

Grygier, M. S., 1994, "Modal Test Technology as Non-Destructive Evaluation of Space Shuttle Structures," in NAS A CP-3263, pp. 329-334.

James, G. H. III, 1996, “Development of Structural Health Monitoring Techniques Using Dynamics Testing," Sandia Report SAND-96-0810.

Juang, J.-N., 1994, Applied System Identification, PTR Prentice-Hall, Inc., Englewood Cliffs, NJ.
Pappa, R. S., Elliott, K. B., and Schenk, A., 1993, "Consistent-Mode Indicator for the Eigensystem Realization Algorithm," Journal of Guidance, Control, and Dynamics, Vol. 16, No. 5, pp. 852-858.

Pappa, R. S., 1994, "Eigensystem Realization Algorithm User's Guide for VAX/VMS Computers," NASA TM-109066.

Pappa, R. S., 1997a, "Eigensystem Realization Algorithm Bibliography," Website: http://sdbwww.larc.nasa.gov/S DB/Research/data/ERA_biblio. html.

Pappa, R. S., Woodard, S. E., and Juang, J.N., 1997b, "A Benchmark Problem for Development of Autonomous Structural Modal Identification," Proceedings of the 15th International Modal Analysis Conference, pp. 1071-1077.

Zimmerman, D. C., Smith, S. W., Kim, H.M., and Bartkowicz, T. J., 1996, "Spacecraft Applications for Damage Detection Using Vibration Testing," Proceedings of the 14 th International Modal Analysis Conference, pp. 851 856. 
Public reporting burden for this collection of intormation is estimated to average 1 hour per response, including the time for reviewing instructions. searching existing data sources. gathering and maintaining the data needed, and completing and reviewing the collection of information. Send comments regarding this burden estimale or any other aspect of this Highway. Suite 1204, Avtington, VA 22202-4302, and to the Office of Management and Budget, Paperwork Reduction Project (0704-0188), Washington, DC 20503.

\begin{tabular}{|l|l|l|}
\hline 1. AGENCY USE ONLY (Leave blank) & $\begin{array}{l}\text { 2. REPORT DATE } \\
\text { June } 1997\end{array}$ & $\begin{array}{l}\text { 3. REPORT TYPE AND DATES COVERED } \\
\text { Technical Memorandum }\end{array}$ \\
\hline
\end{tabular}

4. ITLE AND SUBTTLE
Autonomous Modal Identification of the Space Shuttle Tail
Rudder

Rudder

6. AUTHOR(S)

Richard S. Pappa David C. Zimmerman

George H. James III

7. PERFORMING ORGANIRATION NAME(S) AND ADDRESS(ES)

8. PERFORMING ORGANIPATION REPORT NUMBER

NASA Langley Research Center

Hampton, VA 23681-0001

9. SPONSOAING / MONTORING AGENCY NAME(S) AND ADDRESS(ES)

National Aeronautics and Space Administration

Washington, DC 20546-0001

10. SPONSORING / MONTTORING AGENCY REPORT NUMBER

NASA TM-112866

\section{SUPPLEMENTARY NOTES}

Richard S. Pappa: Langley Research Center, Hampton, VA..; George H. James III and David C. Zimmerman: University of Houston, Houston, TX

Will be presented at the 16th ASME Biennial Conference on Mechanical Vibration and Noise in Sacramento, CA, on September 14-17, 1997

\begin{tabular}{l|l} 
12a. DISTRIBUTHON/AVAILABILTY STATEMENT & 12b. DISTRIBUTION CODE
\end{tabular}

Unclassified - Unlimited

Subject Category 18

Availability: NASA CASI, (301) $621-0390$

13. ABSTRACT (Maximum 200 mords)

Autonomous modal identification automates the calculation of natural vibration frequencies, damping, and mode shapes of a structure from experimental data. This technology complements damage detection techniques that use continuous or periodic monitoring of vibration characteristics. The approach shown in the paper incorporates the Eigensystem Realization Algorithm (ERA) as a data analysis engine and an autonomous supervisor to condense multiple estimates of modal parameters using ERA's Consistent-Mode Indicator and correlation of mode shapes. The procedure was applied to free-decay responses of a Space Shuttle tail rudder and successfully identified the seven modes of the structure below $250 \mathrm{~Hz}$. The final modal parameters are a condensed set of results for 87 individual ERA cases requiring approximately five minutes of CPU time on a DEC Alpha computer.

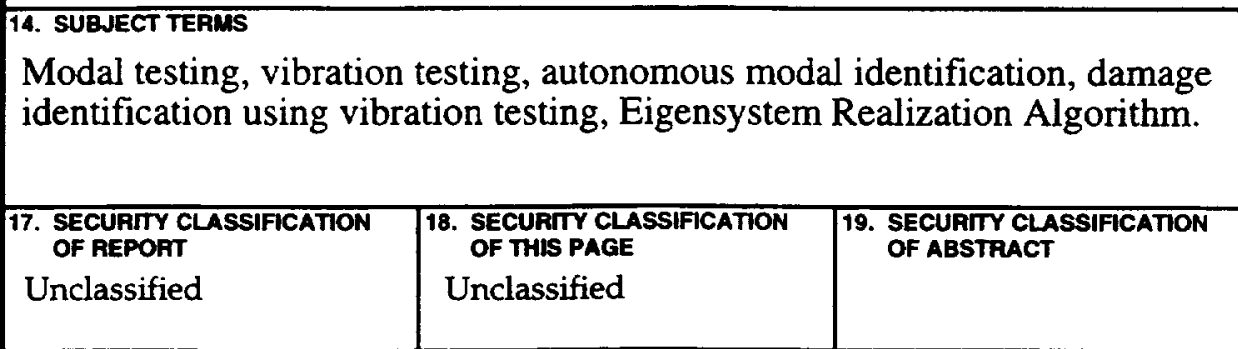

NSN 7540-01-280-5500
15. NUMBER OF PAGES

12

16. PRICE CODE $\mathrm{AO3}$

20. LIMTAATION OF ABSTAACT

Standard Form 298 (Rev, 2-89) Prescribed by ANSI Std. Z39-18 298-102 